\title{
On the validity of different motion capture technologies for the analysis of running*
}

\author{
Frank J. Wouda ${ }^{1}$, Matteo Giuberti ${ }^{2}$, Giovanni Bellusci ${ }^{2}$, Erik Maartens ${ }^{1,3}$, Jasper Reenalda ${ }^{1,3}$, \\ Bert-Jan F. van Beijnum ${ }^{1}$ and Peter H. Veltink ${ }^{1}$
}

\begin{abstract}
An increasing diversity of available motion capture technologies allows for measurement of human kinematics in various environments. However, little is known about the differences in quality of measured kinematics by such technologies. Therefore, this work presents a comparison between three motion capture approaches, based on inertial-magnetic measurement units (processed with Xsens MVN Analyze) and optical markers (processed using Plug-In Gait and OpenSim Gait2392). It was chosen to evaluate the different motion capture approaches in running, as such kinematics are preferably measured in the natural running environment and involve challenging dynamics. An evaluation was done using data of 8 subjects running on a treadmill at three different speeds, namely 10, 12 and $14 \mathrm{~km} / \mathrm{h}$. The sagittal plane results show excellent correlation $(\rho>0.96)$ and RMSDs are smaller than 5 degrees for 6 out of the 8 subjects. However, results in the frontal and transversal planes were less correlated between the different motion capture approaches. This shows that sagittal kinematics can be measured consistently using any of the three analyzed motion capture approaches, but ambiguities exist in the analysis of frontal and transversal planes.
\end{abstract}

\section{INTRODUCTION}

A wide variety of technologies is currently available for capturing three-dimensional full-body human motion, of which the most established ones are based on video or inertial sensors. Optical systems rely on the idea of tracking retroflective markers, placed on pre-defined locations of the different body segments, using infrared cameras [1], [2]. Inertial systems, on the other hand, track the orientation of inertial-magnetic sensors by sensor fusion of the accelerometer, gyroscope and magnetometer output, which can be translated to body segment quantities by using a calibration pose and pre-defined sensor placement on the various body segments [3], [4]. Traditional motion capture was done in a lab via accurate optical systems, while inertial systems were designed to allow for ambulatory measurements, i.e. not restricted to a laboratory setting.

Besides this apparent difference in applicability, an important determinant in the choice for either motion capture technology is the measurement accuracy of parameters of interest. Both inertial-magnetic and optical motion capture

*This research (project No. 13917) is supported by the Dutch Technology Foundation STW, which is part of the Netherlands Organization for Scientific Research (NWO), and which is partly funded by the Ministry of Economic Affairs

${ }^{1}$ Faculty of Electrical Engineering, Mathematics and Computer Science, University of Twente, 7500 AE Enschede, The Netherlands f.j.woudadutwente.nl

${ }^{2}$ Xsens Technologies B.V., Enschede, The Netherlands

${ }^{3}$ Roessingh Research and Development, Roessingh Rehabilitation Hospital, Enschede, The Netherlands accuracy can be influenced by different external factors, e.g. soft-tissue artifacts (STAs) [5], occlusions [6], sensor/marker placement [7], [8], calibration pose accuracy [8], [9] and/or magnetic disturbances [3], [10]. Most of those potential sources of errors can be solved by proper measurement preparation, e.g. camera distribution, marker/sensor placement and clear subject instructions. However, due to differences in sensor/marker placement between protocols and/or technologies, the impact of STAs on the measured kinematics varies [11]. In case of occlusions gap filling can be applied [6], and magnetic disturbances can be mitigated by using advanced sensor fusion techniques [12], [13]. Even though position estimates of retroflective markers are highly accurate (due to cameras with high resolution) [14], estimates of relevant quantities at the body level (e.g., body kinematics) might show a degraded accuracy depending on the considered body modeling assumptions [15] (e.g., Plug-In Gait [16], [17], OpenSim [18] or AnyBody [19]).

Performance comparisons, in terms of kinematics, among different motion capture technologies have been reported in several comparison studies. For example, Stief et. al showed that sagittal plane joint angles in gait measured with Plug-In Gait are more accurate than joint angles in other planes compared to their proposed lower-body marker protocol [20]. An inter-laboratory study showed that sagittal kinematics were consistent between labs and protocols [8]. Comparable results were reported in a comparison between different marker protocols, where out-of-sagittal planes joint angles were shown to have lower correlation for the different marker protocols [21]. Ferrari et al. compared two IMMU with optical protocols and concluded that their protocol can potentially be used for clinical gait assessment [22]. Simarly, a comparison between Plug-In Gait and OpenSim showed that an offset was typically observed between both approaches for walking trials [15]. A musculoskeletal model driven by either IMMU or optical motion capture walking data, showed larger correlations for the sagittal kinematics compared to the frontal and transversal kinematics [23]. Dinu et al. compared centre of mass position estimates during stance and jumps based on IMMU and optical motion capture data, and reported that both estimates are similar and could be used interchangeably [24].

However, to the best of our knowledge, no comparison between different motion capture approaches to assess running kinematics has been performed. Therefore, the aim of this work is to analyze differences in measurement accuracy of different motion capture approaches, namely IMMU, Plug- 
In Gait and OpenSim. Running on a treadmill was chosen as an evaluation scenario due to constraints that a laboratory setting poses on measuring kinematics of runners in their natural running environment [25] (e.g., on the track, road or in the woods) and the more challenging dynamics involved, compared to gait. Even though IMMUs allow for real world measurements, the analysis was performed on a treadmill in a gait lab to allow for simultaneous measurements with optical and inertial-magnetic motion capture.

\section{MATERIALS AND METHODS}

In Section II-A the data collection protocol is introduced. The measurement setup with the different motion capture systems is described in Section II-B. The data processing, including the different motion capture approaches is explained in Section II-C. And the evaluation measures are described in Section II-D.

\section{A. Data collection}

Experimental data was collected in the gait laboratory of Roessingh Research and Development (Enschede, the Netherlands), with approval from the ethics committee of the Faculty of Electrical Engineering, Mathematics and Computer Science at the University of Twente. Eight healthy subjects volunteered for the study (8 males; age: $25.1 \pm$ 5.2 years; height: $183.7 \pm 4.5 \mathrm{~cm}$; weight: $77.7 \pm 9.4 \mathrm{~kg}$; body mass index: $23.0 \pm 2.5 \mathrm{~kg} / \mathrm{m}^{2}$ ). The runners were recruited from a local track \& field club and had no recent (self-reported) history of injuries. After a warm-up session of approximately three minutes at self-selected running speeds, subjects were asked to run for three minutes at three different speeds (namely 10, 12 and $14 \mathrm{~km} / \mathrm{h}$, in this order) on an instrumented treadmill.

\section{B. Measurement setup}

Subjects were equipped with both inertial-magnetic and optical motion capture technologies and they were running on an instrumented treadmill (S-Mill, ForceLink, Culemborg, the Netherlands), with a running area of $250 \times 100 \mathrm{~cm}$. Xsens MVN Link (Xsens, Enschede, the Netherlands) was used to capture full-body movements at $240 \mathrm{~Hz}$ using 17 inertialmagnetic sensors, which were placed in a full-body Lycra suit (as shown in Fig. 1). The suit was adapted in such a way to accommodate placement of 41 retroflective markers on the subject's skin. The position of these markers, placed according to the Plug-in Gait protocol ${ }^{1}$ (Nexus 1.8.5, Vicon, Oxford, UK), was recorded at $100 \mathrm{~Hz}$ by six high-speed infrared cameras (MX-13, Vicon, Oxford, UK).

\section{Data processing}

Only periods of steady state running at a constant speed were included in the analysis. Optical motion capture data (recorded with Vicon) was processed using both Plug-In Gait (a direct kinematics method) [16], [17] and OpenSim (Gait2392, an inverse kinematics method) [18], which for

\footnotetext{
${ }^{1}$ To allow for OpenSim processing, one additional marker was placed on all lower-body segments.
}

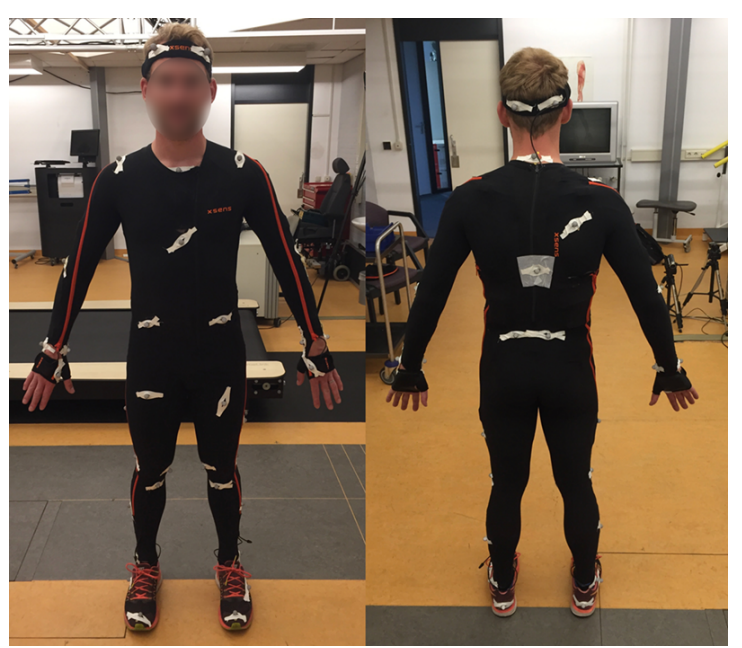

Fig. 1. Placement of the 41 retroflective markers (according to the Plug-In Gait protocol $)^{1}$ can be seen for a representative subject. The markers were placed directly on the subject's skin. IMMU sensors were placed inside a Lycra suit, such that these remain in a fixed position with respect to the body. To accomodate the retroflective markers, holes were cut in this suit. This allowed for simultaneous measurement of kinematics using optical and IMMU motion capture technologies.

simplicity will be referred to as PiG and OS, respectively. IMMU sensor data was processed using MVN Analyze (Xsens, Enschede, the Netherlands), referred to as MVN. These motion capture approaches have been used for analysis of running kinematics in other studies [25], [26], [27], [28], [29], [30]. Kinematic data was assessed in the joint frame, and all approaches use Euler angles in the order of flexion/extension $(\mathrm{F} / \mathrm{E})$, ab-/adduction $(\mathrm{A} / \mathrm{A})$ and internal/external rotation (I/E). For both OS and PiG a static trial was recorded, where the subject performed a neutral pose (standing upright with arms straight next to his/her side) for approximately 5 seconds. The MVN calibration required the same neutral pose, followed by a short walk [13].

Even though full-body kinematics were measured, the analysis was limited to the lower-body outcomes since these are most important in evaluating running kinematics. OS assumes a limited number of degrees of freedom in the knee and ankle joints (modeled as hinges, i.e. only F/E movement is allowed), the knee model of OS was adapted to also allow for A/A and I/E, similar to [13], [31]. In this manner the rotational axes of the adapted OS knee joints aligned with both the PiG and MVN knee joint axes. The transversal and frontal ankle degrees of freedom are modeled in the subtalar joint for OS, and are therefore not modified as these do not align with the ankle joint axes for the PiG and MVN outcomes. All three rotational degrees of freedom are allowed for the lower-body joints for the PiG and MVN models.

For a fair comparison, all data was resampled at 120 $\mathrm{Hz}$ using linear interpolation (upsampling) for the optical data and decimation (downsampling) for the IMMU data. MATLAB R2017a (Mathworks, Inc., Natick, MA, USA) was used for processing all data. 


\section{Evaluation measures}

Pearson's correlation coefficients $(\rho)$ and Root Mean Squared Differences (RMSD) were used to characterize differences in the measured lower-body kinematics of PiG, OS and MVN. This analysis was performed for joint angles in all three planes: sagittal (F/E), frontal (A/A) and transversal (I/E). Segmentation of the kinematic data was required to allow for analysis of individual strides. This segmentation was done by identifying heel strike events using force output of the instrumented treadmill. Mean correlation over all strides was calculated using a Fisher transformation [32]. Possible offsets between the motion capture approaches were evaluated, in which the offset was defined as the mean difference between both outcomes. Furthermore, two relevant discrete outcome measures for running analysis were compared for the different approaches, namely maximum knee $\mathrm{F} / \mathrm{E}$ angle during stance and the ankle $\mathrm{F} / \mathrm{E}$ angle at heelstrike [25], [33].

\section{RESULTS}

Results of the comparison between the three different motion capture approaches are shown in Table I, which is divided according to the planes of movement, namely $\mathrm{F} / \mathrm{E}$ (top), A/A (middle) and I/E (bottom). This table displays the mean correlation coefficient, RMSD (mean and standard deviation over the different strides) and (possible) offset of the measured running kinematics at $12 \mathrm{~km} / \mathrm{h}$. Similar results were obtained for other speeds, but are not shown here due to space limitations. Fig. 2 shows the mean (and standard deviation) joint angle profiles of two representative subjects when running at 12 and $14 \mathrm{~km} / \mathrm{h}$ (shown in Fig. 2A and 2B, respectively).

An excellent correlation $(\rho>0.96)$ between the different approaches was found for all the measured sagittal plane lower-body joint angles (Table I, F/E of the hip, knee and ankle). This can also be seen in Fig. 2, where the largest difference is found in the hip F/E angles.

Fig. 2 shows MVN, PiG and OS give inconsistent joint angle profiles in the frontal (Table I, A/A of the hip, knee and ankle) and the transversal (Table I, I/E of the hip, knee and ankle) planes. The hip A/A angles show largest correlations compared to other joints/planes, where the RMSDs were not larger than 8 degrees between the different approaches. For other joints RMSDs up to 30 degrees were observed, which in some cases (e.g. ankle A/A and I/E) can be largely contributed to an offset between the different approaches.

Boxplots of the measured maximum knee F/E angle during stance and the ankle F/E angle at heelstrike (Fig. 3) show differences of up to 10 degrees between the mean outcomes of the different approaches. Fig. 3A shows that relative differences in the maximum knee $\mathrm{F} / \mathrm{E}$ angle between different approaches are consistent over the various subjects, as in all cases OS shows the largest and MVN the smallest outcomes. Similarly, Fig. 3B shows largest mean ankle F/E angles at heelstrike for PiG and smallest for OS in the majority of the subjects.
TABLE I

INDIVIDUAL RESULTS (EVALUATED AT $12 \mathrm{KM} / \mathrm{H}$, FOR ALL THREE ROTATIONAL AXES: F/E, A/A AND I/E) ARE COMPARED BETWEEN THE DIFFERENT MOTION CAPTURE APPROACHES: MVN, PIG AND OS USING $\rho, \operatorname{RMSD}(\sigma)$ AND OFFSET. NOTE THAT ANKLE A/A AND I/E ARE MISSING FOR OS SINCE THESE ARE NOT ASSESSED IN THE MODEL.

\begin{tabular}{|c|c|c|c|c|c|c|c|c|c|c|}
\hline \multicolumn{2}{|c|}{$\begin{array}{l}\text { Kinematics 1 } \\
\text { Kinematics 2 } \\
\end{array}$} & & $\begin{array}{c}\text { MVN } \\
\text { PiG }\end{array}$ & & & $\begin{array}{l}\text { PiG } \\
\text { OS }\end{array}$ & & & $\begin{array}{c}\text { OS } \\
\text { MVN }\end{array}$ & \\
\hline & & & & Flexi & n/exten & sion angles & & & & \\
\hline & Subjects & $\rho$ & $\operatorname{RMSD}(\sigma)$ & Offset & $\rho$ & RMSD $(\sigma)$ & Offset & $\rho$ & RMSD $(\sigma)$ & Offset \\
\hline & S01 & 0.994 & $8.86(0.76)$ & -8.44 & 0.991 & $2.64(0.39)$ & -0.82 & \begin{tabular}{|l|}
0.986 \\
\end{tabular} & $9.84(0.80)$ & 9.26 \\
\hline & S02 & 0.995 & $11.97(0.78)$ & -11.47 & 0.989 & $4.13(0.35)$ & -2.64 & 0.994 & $14.52(0.45)$ & 14.11 \\
\hline & S03 & 0.993 & $4.02(0.80)$ & -1.02 & 0.989 & $4.83(1.06)$ & -3.91 & 0.992 & $5.60(1.11)$ & 4.93 \\
\hline & S04 & 0.990 & $8.81(0.55)$ & -8.41 & 0.990 & $7.24(1.04)$ & -6.76 & 0.992 & $15.38(1.02)$ & 15.16 \\
\hline 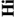 & S05 & 0.986 & $8.91(1.67)$ & -8.24 & 0.992 & $5.87(0.61)$ & -5.31 & 0.985 & $13.99(0.96)$ & 13.55 \\
\hline & S06 & 0.992 & $7.11(1.02)$ & -6.75 & 0.991 & $6.66(0.63)$ & -6.20 & 0.995 & $13.16(0.66)$ & 12.95 \\
\hline & S07 & 0.996 & $12.05(0.93)$ & -11.82 & 0.990 & $6.91(0.69)$ & -6.26 & 0.988 & $18.35(0.37)$ & 18.08 \\
\hline & S08 & 0.990 & $15.30(1.06)$ & -15.06 & 0.990 & $2.97(0.68)$ & -1.70 & 0.984 & $17.03(0.49)$ & 16.76 \\
\hline & \begin{tabular}{|l|} 
Subjects \\
\end{tabular} & $\rho$ & $\operatorname{RMSD}(\sigma)$ & Offset & $\rho$ & RMSD $(\sigma)$ & Offset & $\rho$ & RMSD $(\sigma)$ & Offset \\
\hline & S01 & 0.992 & $7.85(1.31)$ & 6.83 & 0.988 & $7.21(3.93)$ & -5.61 & \begin{tabular}{|l|}
0.985 \\
\end{tabular} & $6.29(0.63)$ & -1.22 \\
\hline & S02 & 0.992 & $7.57(2.82)$ & 5.56 & 0.986 & $7.41(3.98)$ & -5.49 & 0.988 & $6.86(0.79)$ & -0.07 \\
\hline & S03 & 0.986 & $9.93(3.76)$ & 7.65 & 0.985 & $7.09(4.27)$ & -5.48 & 0.984 & $7.15(1.07)$ & -2.18 \\
\hline & S04 & 0.983 & $9.59(1.68)$ & 6.84 & 0.988 & $10.78(5.10)$ & -10.18 & 0.985 & $6.81(1.58)$ & 3.34 \\
\hline 5 & S05 & 0.981 & $10.18(3.04)$ & 7.31 & 0.982 & $8.70(5.50)$ & -7.90 & 0.979 & 7.99 (1.33) & 0.58 \\
\hline & S06 & 0.990 & $8.71(1.71)$ & 7.00 & 0.976 & $9.65(9.51)$ & -7.33 & 0.964 & $8.56(8.74)$ & 0.33 \\
\hline & S07 & 0.992 & $6.65(1.77)$ & 4.20 & 0.985 & $7.48(5.47)$ & -4.31 & 0.987 & $6.39(4.30)$ & 0.12 \\
\hline & S08 & 0.982 & $8.84(2.50)$ & 5.87 & 0.988 & $8.26(4.34)$ & -7.42 & 0.980 & $7.28(1.85)$ & 1.55 \\
\hline & Subjects & $\rho$ & RMSD $(\sigma)$ & Offset & $\rho$ & RMSD $(\sigma)$ & Offset & $\rho$ & RMSD $(\sigma)$ & Offset \\
\hline & S01 & 0.984 & $3.85(1.10)$ & -2.49 & 0.961 & $3.99(0.92)$ & 1.15 & \begin{tabular}{|l|}
$r$ \\
0.971 \\
\end{tabular} & $4.50(1.32)$ & 1.35 \\
\hline & $\mathrm{S} 02$ & 0.975 & $4.00(0.73)$ & -0.85 & 0.967 & $6.01(0.25)$ & 4.62 & 0.978 & $5.28(0.64)$ & -3.77 \\
\hline & S03 & 0.966 & $4.54(1.55)$ & -2.96 & 0.974 & 6.31 & 5.24 & 986 & $3.41(1.19)$ & -2.28 \\
\hline & S04 & 0.981 & $6.87(1.32)$ & -5.88 & 0.968 & 4.75 & 2.38 & .986 & $4.39(0.58)$ & 3.50 \\
\hline & S05 & 0.984 & $4.68(1.22)$ & -3.74 & 0.960 & $4.98(0.16)$ & 3.13 & 0.983 & $3.21(0.83)$ & 0.61 \\
\hline & S06 & 0.975 & $6.24(1.45)$ & -5.42 & 0.968 & $6.49(0.43)$ & 5.57 & 0.976 & $3.03(0.50)$ & -0.16 \\
\hline & S07 & 0.988 & $8.06(1.52)$ & -7.58 & 0.969 & 6.79 & 5.65 & 0.983 & $3.65(1.20)$ & 1.93 \\
\hline & S08 & 0.984 & $3.86(1.06)$ & -2.62 & 0.970 & $4.55(0.18)$ & 3.14 & 0.989 & $2.80(0.33)$ & -0.52 \\
\hline & & & & & adducti & on angles & & & & \\
\hline & Subjects & $\rho$ & RMSD $(\sigma)$ & Offset & $\rho$ & $\operatorname{RMSD}(\sigma)$ & Offset & $\bar{\rho}$ & RMSD $(\sigma)$ & Offset \\
\hline & So1 & 0.823 & $4.25(0.96)$ & 0.10 & 0.761 & $8.38(4.32)$ & -3.50 & \begin{tabular}{|l|}
0.904 \\
\end{tabular} & $2.98(0.29)$ & 1.92 \\
\hline & $\mathrm{S} 02$ & 0.757 & 5.44 & -2.04 & 0.48 & & -1.6 & 633 & & -0.05 \\
\hline & S03 & 0.683 & $2.76(0.89)$ & -0.63 & 0.587 & $3.98(1.05)$ & -0.63 & 0.467 & $3.36(0.31)$ & 0.92 \\
\hline & S04 & 0.672 & $4.72(0.44)$ & 0.60 & 0.689 & $7.32(1.06)$ & 4.13 & 0.747 & $3.79(0.65)$ & 2.58 \\
\hline & S05 & 0.391 & $6.06(0.87)$ & 0.52 & 0.868 & 5.24 & 0.13 & .568 & $3.84(0.28)$ & 1.52 \\
\hline & S06 & 0.772 & $4.04(1.15)$ & -2.95 & 0.814 & $5.98(2.05)$ & -2.05 & .935 & $3.79(2.04)$ & 3.22 \\
\hline & S07 & 0.666 & $5.10(0.28)$ & 0.28 & 0.743 & $5.80(2.45)$ & -2.45 & 0.837 & $4.32(0.87)$ & 3.66 \\
\hline & S08 & 0.299 & $4.84(1.13)$ & 1.13 & 0.640 & $5.18(1.80)$ & -1.80 & 0.765 & $3.27(0.29)$ & 1.78 \\
\hline & Subjects & $\rho$ & $\operatorname{RMSD}(\sigma)$ & Offset & $\rho$ & $\operatorname{RMSD}(\sigma)$ & Offset & $\rho$ & $\operatorname{RMSD}(\sigma)$ & Offset \\
\hline & S01 & 0.637 & $4.77(1.31)$ & 2.76 & 0.341 & $10.77(3.26)$ & -6.51 & \begin{tabular}{|l|}
0.072 \\
\end{tabular} & $10.33(3.92)$ & 4.15 \\
\hline & S02 & 0.876 & & -2.32 & 0.511 & & -2 & 392 & & \\
\hline & S03 & 0.708 & $16.44(3.76)$ & -8.83 & 0.484 & $17.91(0.94)$ & 0.03 & 0.351 & $13.01(1.61)$ & 0.79 \\
\hline & S04 & 0.372 & 8.89 (1.68) & -6.66 & 0.542 & $12.81(6.20)$ & -8.70 & 312 & $12.90(0.63)$ & -10.09 \\
\hline & S05 & 95 & 6. & 2.90 & 0.546 & & -5 . & 398 & 12.4 & 8.69 \\
\hline & S06 & 0.244 & $8.29(1.75)$ & -3.14 & 0.503 & $16.90(5.57)$ & -13.55 & 0.228 & $11.43(2.44)$ & 0.16 \\
\hline & S07 & 0.532 & $11.67(1.77)$ & -7.85 & 0.637 & $12.16(6.64)$ & 0.31 & 0.500 & $11.18(3.64)$ & 4.89 \\
\hline & S08 & 0.347 & $8.03(2.50)$ & -3.66 & 0.150 & 10.6 & -1.00 & 0.340 & $11.32(0.78)$ & 8.06 \\
\hline & Subjects & $\rho$ & RMSD $(\sigma)$ & Offset & $\rho$ & RMSD $(\sigma)$ & Offset & $\rho$ & RMSD $(\sigma)$ & Offset \\
\hline & S01 & 0.716 & $9.75(1.28)$ & 6.50 & & & & & & \\
\hline & $\mathrm{S} 02$ & 0.518 & & & & & & & & \\
\hline & S03 & 91 & & 29 & & & & & & \\
\hline & S04 & & 12 & 5. & & & & & & \\
\hline & S05 & 0.649 & & 1. & & & & & & \\
\hline & S06 & 67 & 11 & -2.3 & & & & & & \\
\hline & S07 & & & 12 & & & & & & \\
\hline & S08 & 0.595 & $14.55(6.20)$ & 12.82 & & & & & & \\
\hline & & & & Internal/ & ternal & rotation angle & & & & \\
\hline & Subjects & $\rho$ & RI & Offset & & & Offset & $\rho$ & $\mathrm{D}(\sigma)$ & Offset \\
\hline & S01 & 0.287 & $8.25(0.67)$ & -3.1 & $\frac{r 248}{0.9}$ & & -5.96 & 213 & $12.83(3.72)$ & 0.46 \\
\hline & S02 & 0.119 & & & & & & 6 & 16.78 & -6.07 \\
\hline & S03 & 0.134 & $6(8.21)$ & -9.46 & 83 & $20.77(6.31)$ & 12.59 & 102 & $13.28(3.73)$ & -3.13 \\
\hline & S04 & 0.236 & & 13.31 & 0.722 & & 8.56 & 0.139 & 13.2 & -1.23 \\
\hline & S05 & 0.442 & & -0.9 & 0566 & & -2.4 & .535 & 14. & 3.37 \\
\hline & S06 & & 23 & -22.96 & 97 & & -26.31 & 7 & 14.45 & 3.37 \\
\hline & S07 & 0.042 & & 3.66 & 0.366 & & 0.4 & 77 & & 8.03 \\
\hline & S08 & 0.369 & 12.59 & -9.91 & 0.177 & 14.7 & 7.3 & 0.456 & $12.88(1.97)$ & 6.86 \\
\hline & Subjects & - & $\operatorname{RMSD}(\sigma)$ & Offset & & $\mathrm{RM}$ & Offset & $\rho$ & RMSD $(\sigma)$ & Offset \\
\hline & S01 & 0.142 & & -3.4 & & & 9.3 & 7 & & 3.09 \\
\hline & S02 & & & & & & 18. & 1 & & \\
\hline & S03 & 0.237 & $16.44(9.48)$ & -15.58 & 0.327 & 22.3 & 17.05 & .372 & 13.51 & -1.48 \\
\hline & S04 & 0.312 & & -10.40 & 0.514 & & 7.7 & 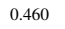 & & 2.6 \\
\hline & S05 & 0 & & 0.4 & 0.5 & & 1.8 & 0 & & -2 \\
\hline & S06 & 0.676 & $8.29(3.26)$ & -16.43 & 0.630 & $19.73(5.57)$ & 15.87 & 0.352 & $16.62(2.44)$ & 0.56 \\
\hline & S07 & 0.220 & & 7.2 & 0.697 & & 26.60 & .365 & & -4.38 \\
\hline & S08 & 0.446 & & 2.1 & 0.490 & & 13.40 & 0.441 & 12.27 & -1.77 \\
\hline & Subjects & $\rho$ & $\operatorname{RMSD}(\sigma)$ & Offset & $\rho$ & $\operatorname{RMSD}(\sigma)$ & Offset & $\rho$ & RMSD $(\sigma)$ & Offset \\
\hline & so1 & 0.08 & & & & & & & & \\
\hline & So & & & & & & & & & \\
\hline & $\mathrm{S} 03$ & 0.143 & $13.45(8.85)$ & & & & & & & \\
\hline & S04 & 0. & & 7.7. & & & & & & \\
\hline & S05 & & & -6. & & & & & & \\
\hline & sot & & $4.11(0.62)$ & -0 . & & & & & & \\
\hline & S07 & 0. & $7.24(0$. & & & & & & & \\
\hline & S08 & 0.426 & $5.63(0.96)$ & -4.63 & & & & & & \\
\hline
\end{tabular}

\section{DISCUSSION}

The results show excellent correlations $(\rho>0.96)$ for the sagittal plane joint angles (Table I, top part), while the frontal and transversal planes show larger differences between the 
different motion capture approaches, also when based on the same technology (optical motion capture, processed with PiG and OS). This is a relevant outcome as for both the knee and ankle joints the main axes of movement is F/E during running; however, other axes are also considered important for assessment of running kinematics [34]. Soft-tissue artifacts (STAs) can be a source of observed differences between the different technologies (optical and inertial-magnetic motion capture), but not for the differences in kinematics obtained by processing with PiG or OS [11]. It has been shown that STA have the smallest impact in the sagittal plane [35]; however, such STA effects can be further reduced by combining video with IMMU information [36]. Another factor mostly impacting non-sagittal planes is the potential misalignment of actual joint axes with the ones in the biomechanical model [37]. This misalignment then results in interference between the different axes of rotation. A dynamic calibration can minimize such misalignment effect, as was performed for the IMMU processing [13].

Table I reports offsets for most of the joints; however, it should be noted that when correlations are weak, RMSD between the approaches cannot be solely attributed to the calculated offsets. On the other hand, the hip F/E offset can be mostly contributed to an offset between different approaches (MVN, PiG and OS) as it is of similar size as the RMSD and correlations are excellent $(\rho>0.98)$. MVN shows the largest offset compared to both $\mathrm{PiG}$ and OS, since this offset is observed consistently for all measured subjects, it is likely caused by the calibration procedure [8], [9]. Pelvic tilt can differ between subjects in the calibration pose while identical assumptions about that pose are still applied to each subject. A knee F/E angle offset of 4.8 degrees was reported by Lathrop et al. between $\mathrm{OS}$ and $\mathrm{PiG}$, and similar results (6.7 degrees on average) were found in this work as shown in Table I [15].

Observed differences in ankle $\mathrm{F} / \mathrm{E}$ angles at heelstrike (Fig. 3B) could result in inconsistent categorization of runner's phenotypes based on their landing pattern [33]. More generally, this may translate to interpretation of general gait classifications, such as disease severity [38]. Due to differences of up to 10 degrees for both discrete outcomes it is difficult to compare such outcomes fairly between the different approaches. However, the observed offsets (Table I) in sagittal knee and ankle joints could have contributed to these differences in maximum knee $\mathrm{F} / \mathrm{E}$ during stance and ankle F/E angle at heelstrike. Furthermore, due to the consistency of relative differences over the various measured subjects, such differences between approaches can potentially be compensated for.

Even though differences in the frontal and transversal planes are larger than in the sagittal plane, observed (in Fig. 2) joint angle profiles in those planes were similar over the various subjects and speeds within each motion capture approach. To assess consistency of the frontal and transversal planes more work should be done to also take into account marker placement variability. Therefore, comparing motion capture data from various approaches in non-sagittal planes can result in inconsistent interpretations and conclusions about the measured kinematics.

\section{CONCLUSIONS}

In this work a comparison between three different motion capture protocols was presented for the analysis of running kinematics, namely based on IMMUs (MVN) and optical markers (PiG and OS). Joint angles in the sagittal plane were measured with the largest similarity in terms of high correlation coefficients between all three approaches. However, differences between the approaches existed and were larger in the frontal and transversal planes than for the sagittal plane, which could lead to inconsistent interpretations. Unlike general opinion which labels optical systems as the golden standard for motion capture, this work shows that clear disagreement between optical kinematic data processed with $\mathrm{PiG}$ and $\mathrm{OS}$ is present in the frontal and transversal planes, while consistent temporal behavior patterns were found in the sagittal plane. Therefore, the choice of one motion capture approach over the other ones will impact results and may impact interpretation of the measured phenomena. And hence given the importance of measuring running in a natural environment, using an IMMU system can be an equally reasonable alternative to optical motion capture systems for sagittal analyses.

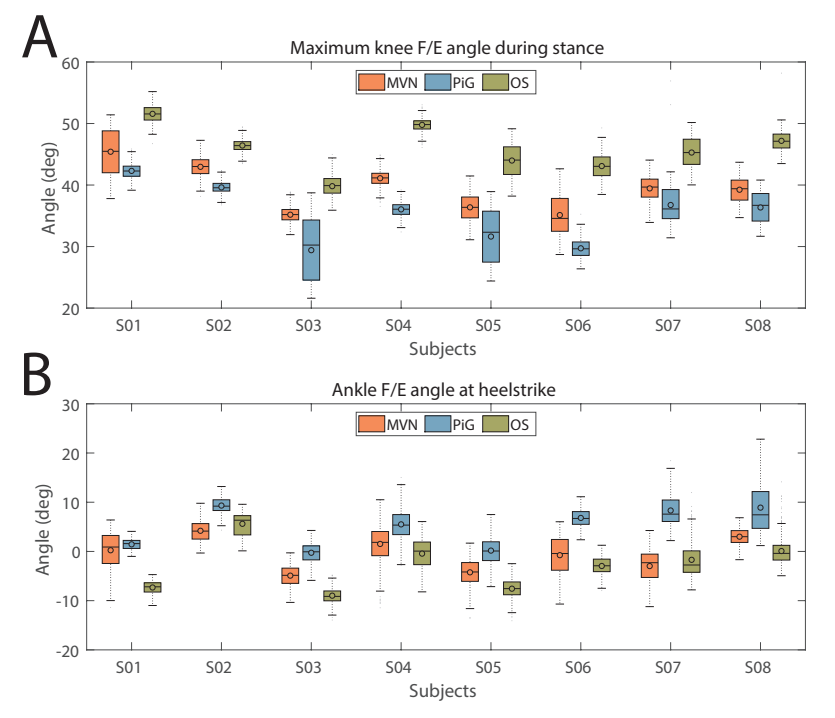

Fig. 3. Comparison between discrete running outcomes (at $12 \mathrm{~km} / \mathrm{h}$ ) of all measured subjects from three motion capture approaches: MVN (orange), $\mathrm{PiG}$ (blue) and OS (green). A shows the maximum knee F/E angle during stance, and $\mathbf{B}$ shows the ankle F/E angle at heel strike. These box plots were obtained from approximately 400 strides.

\section{ACKNOWLEDGMENT}

The authors would like to thank Roessingh Research \& Development (Leendert Schaake in particular) for providing access to the gait laboratory and help with processing the optical motion capture data. 


\section{REFERENCES}

[1] Vicon, http://www.vicon.com/, Accessed on January 292018.

[2] Qualisys, http://www.qualisys.com/, Accessed on January 292018.

[3] D. Roetenberg, H.J. Luinge, and P. Slycke, "Xsens MVN : Full 6DOF Human Motion Tracking Using Miniature Inertial Sensors.," Xsens Technologies, pp. 1-9, April 2013.

[4] Xsens Technologies B.V., https://www.xsens.com/, Accessed on January 292018

[5] A. Leardini, L. Chiari, U. Della, and A. Cappozzo, "Human movement analysis using stereophotogrammetry Part 3. Soft tissue artifact assessment and compensation.," Gait \& Posture, vol. 21, no. 2, pp. 212-225, 2005.

[6] X. Chen and J. Davis, "Camera Placement Considering Occlusion for Robust Motion Capture", Technical Report CS-TR-2000-07, Stanford University, December 2000.

[7] J.L. McGinley, R. Baker, R. Wolfe, and M.E. Morris, "The reliability of three-dimensional kinematic gait measurements: A systematic review.," Gait \& Posture, vol. 29, no. 3, pp. 360-369, 2009.

[8] M.G. Benedetti, A. Merlo, and A. Leardini, "Inter-laboratory consistency of gait analysis measurements.," Gait \& Posture, vol. 38, no. 4, pp. 934-939, 2013.

[9] X. Robert-Lachaine, H. Mecheri, C. Larue, and A. Plamondon, "Accuracy and repeatability of single-pose calibration of inertial measurement units for whole-body motion analysis.," Gait \& Posture, vol. 54, pp. 80-86, 2017.

[10] W.H.K. de Vries, H.E.J. Veeger, C.T.M. Baten, and F.C.T. van der Helm, "Magnetic distortion in motion labs, implications for validating inertial magnetic sensors.," Gait \& Posture, vol. 29, no. 4, 535-541, 2009.

[11] A. Cereatti, T. Bonci, M. Akhbarshahi, K. Aminian, A. Barr, M. Begon, D.L. Benoit, C. Charbonnier, F. Dal Maso, S. Fantozzi, C. Lin, T. Lu, M.G. Pandy, R. Stagni, A.J. van den Bogert, and V. Camomilla "Standardization proposal of soft tissue artefact description for data sharing in human motion measurements.," Journal of Biomechanics, vol. 62, pp. 5-13, 2017.

[12] M. Kok, J.D. Hol, and T.B. Schn, "An optimization-based approach to human body motion capture using inertial sensors.," in IFAC Proceedings Volumes, vol. 47, no. 3, pp. 79-85, 2014.

[13] M. Schepers, M. Giuberti, and G. Bellusci, "Xsens MVN: Consistent Tracking of Human Motion Using Inertial Sensing." Xsens Technologies, pp. 1-8, 2018.

[14] P. Merriaux, Y. Dupuis, R. Boutteau, P. Vasseur, and X. Savatier, "A study of vicon system positioning performance.," Sensors, vol. 17, no. 7, pp. 1-18, 2017.

[15] R.L. Lathrop, A.M.W. Chaudhari, and R.A. Siston, "Comparative Assessment of Bone Pose Estimation Using Point Cluster Technique and OpenSim.," Journal of Biomechanical Engineering, vol. 133, no, 11, pp 1-2, 2011.

[16] R.B. Davis, S. Ounpuu, D. Tyburski, and J.R. Gage, "A gait analysis data collection and reduction technique.," Human Movement Science, vol. 10, no. 5, pp 575-587, 1991

[17] M.P. Kadaba, H.K. Ramakrishnan, and M.E. Wootten, "Measurement of lower extremity kinematics during level walking.," Journal of Orthopedic Research, vol. 8, no. 3, pp. 383-392, 1990.

[18] S.L. Delp, F.C. Anderson, A.S. Arnold, P. Loan, A. Habib, C.T. John, E. Guendelman, and D.G. Thelen, "OpenSim : Open-Source Software to Create and Analyze Dynamic Simulations of Movement.," IEEE Transactions on Biomedical Engineering, vol. 54, no. 11, pp. 19401950, 2007.

[19] M. Damsgaard, J. Rasmussen, S.T. Christensen, E. Surma, and M. de Zee, "Analysis of musculoskeletal systems in the AnyBody Modeling System.," Simulation Modelling Practice and Theory, vol. 14, no. 8, pp. 1100-1111, 2006.

[20] F. Stief, H. Bhm, K. Michel, A. Schwirtz, and L. Dderlein, ’Reliability and Accuracy in Three-Dimensional Gait Analysis : A Comparison of Two Lower Body Protocols.," Journal of Applied Biomechanics, vol. 29 , no. 1, pp. 105-111, 2013.

[21] A. Ferrari, M.G. Benedetti, E. Pavan, C. Frigo, D. Bettinelli, M. Rabuffetti, P. Crenna, and A. Leardini, "Quantitative comparison of five current protocols in gait analysis.," Gait \& Posture, vol. 28, no. 2, pp. 207-216, 2008.

[22] A. Ferrari, A.G. Cutti, P. Garofalo, M. Raggi, M. Heijboer, A. Cappello, and A. Davalli, "First in vivo assessment of ?outwalk?: A novel protocol for clinical gait analysis based on inertial and magnetic sensors.," Medical \& Biological Engineering \& Computing, vol. 48, no. 1 , pp. 1-15, 2010.

[23] A. Karatsidis, M. Jung, H.M. Schepers, G. Bellusci, M. de Zee, P.H. Veltink, and M.S. Andersen, "Predicting kinetics using musculoskeletal modeling and inertial motion capture.," arXiv:1801.01668, 2018.

[24] D. Dinu, M. Fayolas, M. Jacquet, E. Leguy, J. Slavinski, and N. Houel, "Accuracy and reliability of postural human-motion tracking using miniature inertial sensors.," Procedia Engineering, vol. 147, pp. 655658, 2016.

[25] J. Reenalda, E. Maartens, L. Homan, and J.H. Buurke, "Continuous three dimensional analysis of running mechanics during a marathon by means of inertial magnetic measurement units to objectify changes in running mechanics.," Journal of Biomechanics, vol. 49, no. 14, pp. 3362-3367, 2016.

[26] C. Strohrmann, H. Harms, G. Troster, S. Hensler, R. Muller, "Out of the lab and into the woods: kinematic analysis in running using wearable sensors.," Proceedings of the 13th International Conference on Ubiquitous Computing, pp. 119-122, 2011

[27] S.R. Hamner, A. Seth, and S.L. Delp, "Muscle contributions to propulsion and support during running.," Journal of Biomechanics, vol. 43, no. 14, pp. 2709-2716, 2010.

[28] T.W. Dorn, A.G. Schache, and M.G. Pandy. "Muscular strategy shift in human running: dependence of running speed on hip and ankle muscle performance.," Journal of Experimental Biology, vol. 215, no. 11, pp. 1944-1956, 2012.

[29] P.O. Riley, J. Dicharry, J. Franz, U.D. Croce, R.P. Wilder, and D.C. Kerrigan, "A kinematics and kinetic comparison of overground and treadmill running.," Medicine and Science in Sports and Exercise, vol. 40, no. 6, pp. 1093-1100, 2008.

[30] J.P. Kulmala, J. Avela, K. Pasanen, and J. Parkkari, "Forefoot strikers exhibit lower running-induced knee loading than rearfoot strikers.,' Medicine and Science in Sports and Exercise, vol. 45, no. 12, pp. 2306-2313, 2013.

[31] C. Pizzolato, M. Reggiani, D.J. Saxby, E. Ceseracciu, L. Modenese, and D.G. Lloyd, 'Biofeedback for Gait Retraining Based on Real-Time Estimation of Tibiofemoral Joint Contact Forces.," IEEE Transactions on Neural Systems Rehabilitation Engineering, vol. 25, no. 9, pp. 1612-1621, 2017

[32] D.M. Corey, W.P. Dunlap, and M.J. Burke, ”Averaging Correlations: Expected Values and Bias in Combined Pearson rs and Fisher's z Transformations.," The Journal of General Psychology, vol. 125, no. 3, pp. 245-261, 1998.

[33] B. Breine, P. Malcolm, I. Van Caekenberghe, P. Fiers, E.C. Frederick and D. De Clercq, "Initial foot contact and related kinematics affect impact loading rate in running", Journal of Sports Sciences, vol. 35, no. 15 , pp. 1556-1564, 2017.

[34] T.F. Novacheck, "The biomechanics of running.," Gait \& Posture, vol. 7, no. 1, pp. 77-95, 1998.

[35] R. Stagni, S. Fantozzi, A. Cappello, and A. Leardini, "Quantification of soft tissue artefact in motion analysis by combining 3D fluoroscopy and stereophotogrammetry: A study on two subjects.," Clinical Biomechanics, vol. 20, no. 3, pp. 320-329, 2005.

[36] M.C. Bisi, R. Stagni, A. Caroselli, and A. Cappello, "Anatomical calibration for wearable motion capture systems: Video calibrated anatomical system technique.," Medical Engineering \& Physics. vol. 37, no. 8, pp. 813?819, 2015.

[37] T.F. Besier, D.L. Sturnieks, J.A. Alderson, and D.G. Lloyd, ”Repeatability of gait data using a functional hip joint centre and a mean helical knee axis.," Journal of Biomechanics, vol. 36, no. 8, pp. 1159-1168, 2003.

[38] M. Ferrarin, G. Bovi, M. Rabuffetti, P. Mazzoleni, A. Montesano, E. Pagliano, A. Marchi, A. Magro, C. Marchesi, D. Pareyson, and I. Moroni, "Gait pattern classification in children with Charcot-MarieTooth disease type 1A" Gait \& Posture, vol. 35, no. 1, pp. 131-137, 2012. 
- MVN - PiG - OS

Hip F/E
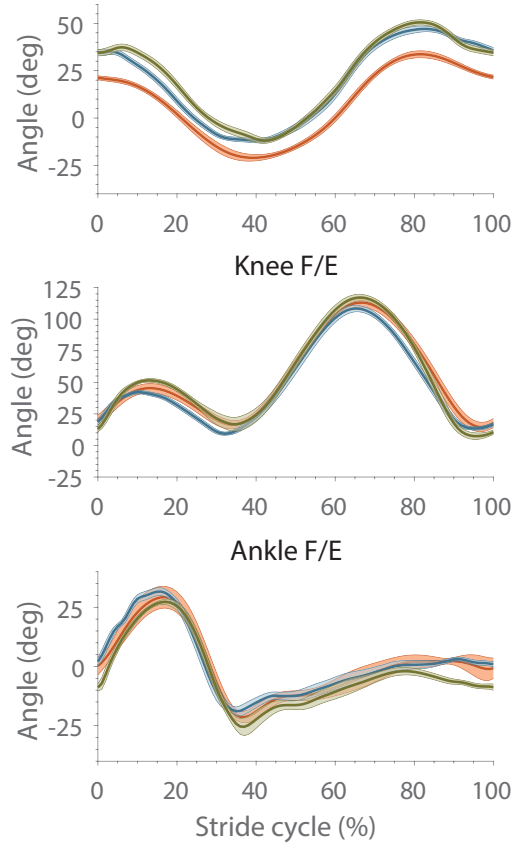

B
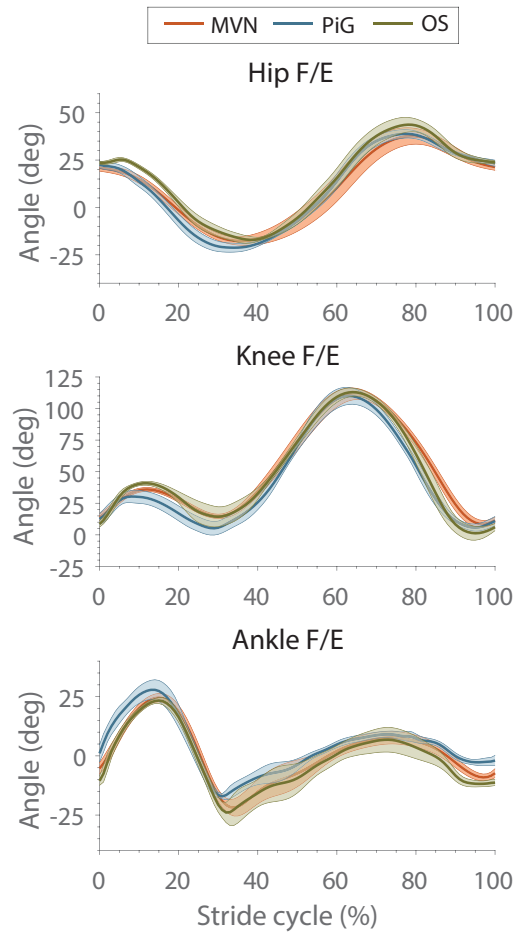

Subject $2(12 \mathrm{~km} / \mathrm{h})$

Hip A/A
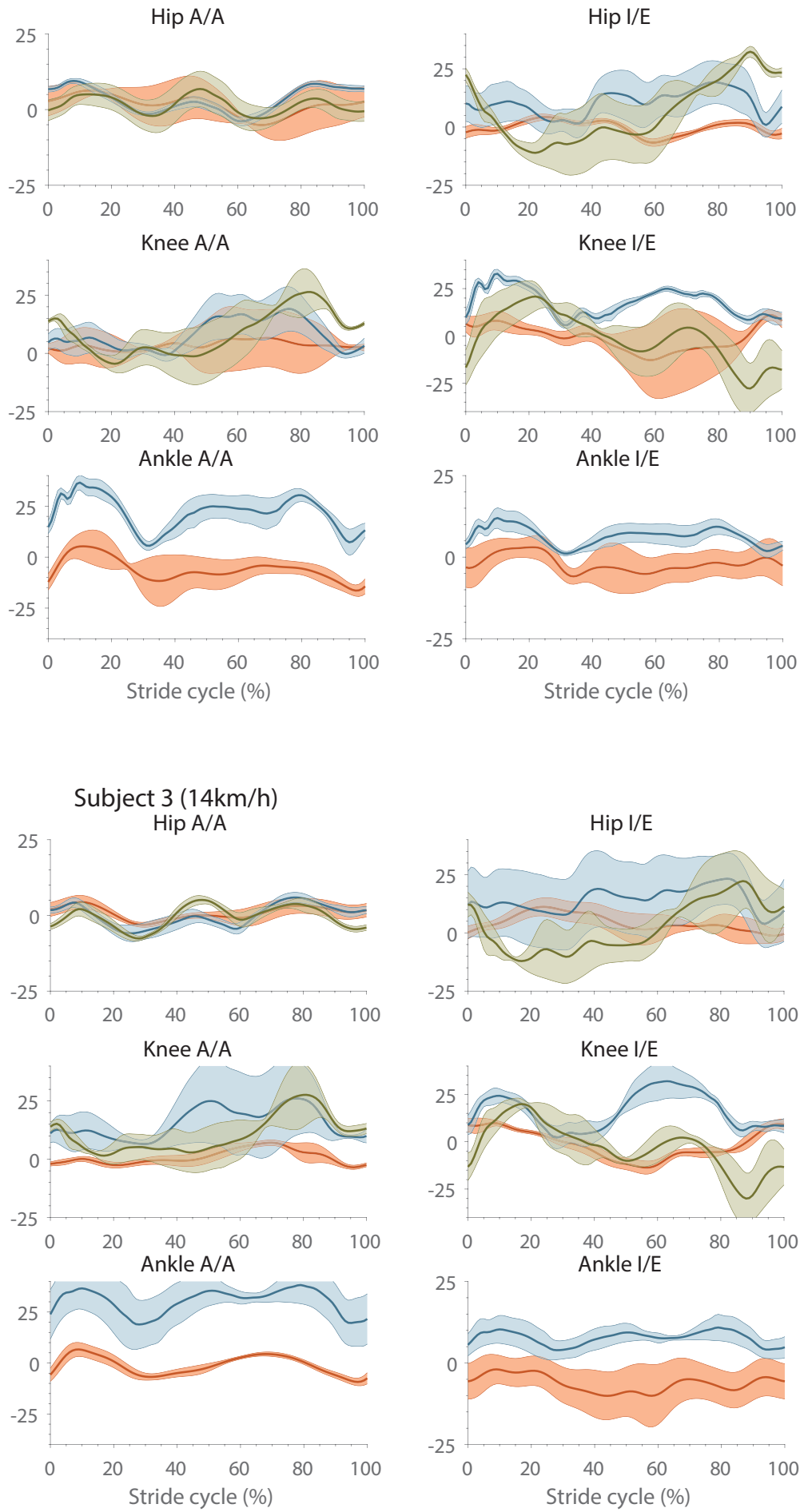
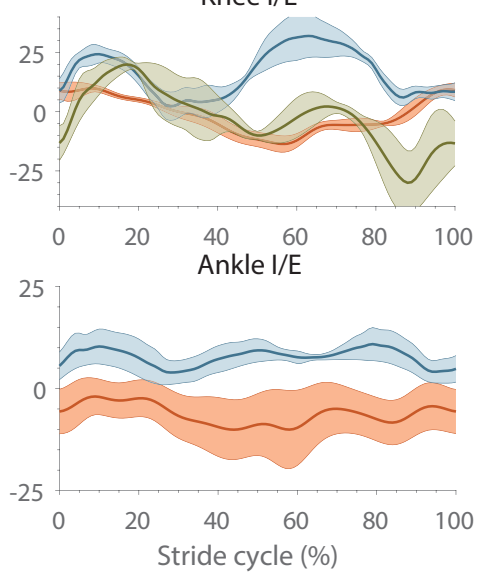

Fig. 2. Comparison between running kinematics of two representative subjects (A shows subject 2 at $12 \mathrm{~km} / \mathrm{h}$ and $\mathbf{B}$ shows subject 3 at $14 \mathrm{~km} / \mathrm{h}$ ) obtained from three motion capture approaches: MVN (orange), PiG (blue) and OS (green). Angles are displayed as a mean (and standard deviation band) normalized to the stride cycle. The left column shows flexion/extension (F/E), in the middle ab-/adduction (A/A) and the right column internal/external (I/E) rotation for the hip, knee and ankle joints. These results are obtained from approximately 400 strides. Corresponding correlations, RMSD (standard deviation) and offset can be found in Table I. 